\title{
Performance Review of MIMO-WiMAX
}

\author{
Akanksha Sharma and Lavish Kansal* \\ School of Electronics and Electrical Engineering, Lovely Professional University, \\ Phagwara - 144411, Punjab, India; \\ akkugood.sharma@gmail.com, lavish.15911@|pu.co.in
}

\begin{abstract}
Orthogonal Frequency Division Multiplexing (OFDM) is mostly considered because of its highly efficient bandwidth capability, its high data rate and ability to prevent multipath fading. It is much suitable technique for parallel transmission and use Wireless interoperability for Microwave Access (WiMAX) on its physical layer. Higher the speed of communication better will be the veracity in it. This broadband is trying its best to get available to every place where they are lagging behind and should be accessible to all as soon as possible. On the other hand, Multiple Input Multiple Output (MIMO) techniques are important part of IEEE 802.16e 2005 specifications in adaptable WiMAX systems. From WiMAX technology, we can conquer the current problems of mobile communication systems like precise coverage of area, flat data rate and dearth of security. In this paper, the focus is on WiMAX model along with MIMO techniques. Moreover, ideas regarding MIMO-WiMAX are also highlighted.
\end{abstract}

Keywords: MIMO, OFDM, SD, SM, WiMAX

\section{Introduction}

An IEEE 802.16 standard based technology WiMAX is positioned on Broadband Wireless Access (BWA). The IEEE 802.16e air interface standard depends on technology named, OFDM, to mitigate the Inter-Symbol Interference (ISI) over delay spread for the wideband wireless networks. The WiMAX standard 802.1 6e supports firm, roving, handy and versatile wireless wideband connection without the need of straight shot with the base station. This WiMAX is different with earlier ones by its mobility. OFDM is a digital transmission method developed to transmit high rate streams using multiple subcarriers called as multicarrier modulation. It converts incomplete revoke of radio signals into small flat fading channels. It is an extraordinary part of OFDM, where carrier frequencies have no relation in them. Here subcarrier spacing is equal to the reciprocal of symbol time (amount of time that transmitter spends performing Inverse Fast Fourier Transform (IFFT)). Adding MIMO feature is mainly to improve the performance of WiMAX system and its capacity too. MIMO is required mainly for efficient communication as using one antenna at both the ends do not work, so, we move for multiple antennas called as MIMO. By transferring absolute information from the specific antennas, we get spatial multiplexing in MIMO systems.

The remaining paper is catalogued as follows: Section 2 is a literature survey about the MIMO-WiMAX systems. Section 3 introduces the detailed the description of OFDM as physical layer of WiMAX contemplated in the paper. Then MIMO systems are defined with their classifications and concepts in Section 4. Section 5 incorporates brief introduction to MIMO-WiMAX system. Finally, conclusions are inferred in Section 5.

${ }^{*}$ Author for correspondence 


\section{Literature Review}

MIMO communication achieves high data rates than wireless channels. The large potential flexibility and adaptively of OFDM transmission technique in frequency selective and time variant radio channels are described by comparing different demodulations methods and channel coding with soft decision decoding and multilevel coding for improving BER performance ${ }^{1}$. Maximum likelihood estimation of OFDM carrier frequency offset in the presence of virtual carriers is considered and showed that it extends to cases involving multiple blocks on comparing with music like algorithm ${ }^{2}$.

For Bit Error Rate (BER) performance and output correlation function of non-linear device, analytical expressions are obtained and $\mathrm{AM} / \mathrm{AM}$ and $\mathrm{AM} / \mathrm{PM}$ non-linearity's represented by Bessel series expansion are derived 3 . On comparing performances of Wi-fi and WiMAX technology by including issues likes security, seamless handover, locate on and emergency services, cooperation and QoS etc. along with their performances which showed WiMAX is more secure and reliable ${ }^{4}$. To understand the basic RS code techniques, modulation performance, cyclic prefix tasks and factors and OFDM symbol on WiMAX PHY layer is studied. Use of Reed Solomon Encoding and Convolution Encoding is also encountered to analyze BER vs. SNR 5 . The effect of Block Interleaving on BER performance of WiMAX PHY layer is considered. Using digital modulation schemes which showed improvement in FEC system performance by reducing burst errors during transmission $\underline{6}$. The use of different channel equalizers and with STBC is introduced and analyzed on WiMAX PHY layer. Results showed error free transmission in it $^{7}$. To improve the problems of $802.16 \mathrm{e}$ protocol, $802.16 \mathrm{~g}$ protocol is introduced. They equated working principles of $802.16 \mathrm{e}$ protocol and $802.16 \mathrm{~g}$ protocol and used technologies like OFDM, MIMO, SC-FDM which improves spectral efficiency of frequency band ${ }^{8}$. Introduction of two MIMO profiles (Alamouti's STC and $2^{\star} 2$ spatial Multiplexing scheme) and compared them. They evaluated their performance with ITU conventional B channel model with $3 \mathrm{~km} / \mathrm{hr}$ speed of pedestrian and revealed BER of $10^{\wedge}-4$ of two MIMO systems give rise to same performance. At inferior
BER values, Matrix A and B gives suitable performances? The comparison of MIMO with SISO is performed on adding Singular Value Decomposition (SVD) in MIMO and channel capacity is developed which resulted in high throughput of SISO and carrying high traffic load by MIMO $\stackrel{10}{ }$.

Obstacles like important feedback aloft, performance deterioration due to feedback delay and huge storage necessity at mobile devices prevents precoding from wide deployment. On adding fifteen antenna/feedback configurations to vector-based, structured codebooks with low intricacy is proposed ${ }^{11}$. To improve BER performance it is analyzed that under obstruction, the channel capacity of intended MIMO system is high than traditional systems and they are based on V-BLAST having two schemes i.e. Spectrum Domain Encoding (SDE) and Wavelet Domain Encoding (WDE) ${ }^{12}$. A transmission scheme is developed to eliminate channel distortion to improve noise immunity and BER. Various Transmit Diversity schemes and Diversity combining techniques are compared by simulations ${ }^{13}$. Various methods to evaluate MIMO-WiMAX in multi cell, multi sector and multi user environment for single frequency reuse. They used both uplink and downlink where SM of downlink show 10\% improvement over SIMO and uplink increase the spectral efficiency by $9 \%$ over SIMO system same as beamforming $\frac{14}{}$. Two MIMO PHY layer modifications (STBC) and (SM) are investigated and provided high bandwidth effectiveness, turnout and data rates. With the help of MATLAB simulations they obtained mechanisms to improve WiMAX PHY layer with high spectral efficiency ${ }^{15}$. To study time synchronization errors in OFDM system, its major effects, performances with BER and compared with some synchronization algorithms with OFDM system which showed fading environment and gives good picture of practical scenario $\frac{16}{6}$. Proposed method of parallel combination scheme to reduce PAPR in MIMO-OFDM system below Rayleigh fading environment which reduces PAPR and complexity of system and maintain BER also ${ }^{17}$. Effects, estimation and correction of frequency offsets are done along with MIMO-OFDM combinations and techniques in presence of CFO. Comparison is done between $2 * 1$ STBC-OFDM, $2 * 2$ STBC-OFDM, $2^{\star} 1$ SFBC-OFDM 
and gave better results. Diversity is also adversely affected by $\mathrm{CFO}^{18}$.

Introduction of real MIMO-WiMAX platform to simulate space, time and frequency selective fading conditions and used this platform to simulate Space-Time Codes (STC), Space-Frequency Codes (SFC) and SpaceTime-Frequency Codes (STFC) where STFC showed multiplicity gain compared to STC and SFC ${ }^{19}$. Downlink performance of two full-rate STC's of IEEE 802.16e i.e. Matrix B and Matrix C which showed Matrix c has long and complex decoding and least used in current WiMAX system $^{20-22}$. Using multiple antenna options and comparing their performance we got same spectral efficiency, Matrix A perform better than Matrix B and throughput is maximized by combining MIMO with adaptive modulation and coding 23,24 . Measurement of turnout is done to compare with channel capability and shared information which obtain SNR detriment at turnout of $5 \mathrm{Mbits} / \mathrm{s}$ to 6 $\mathrm{db}$ through channel code and channel estimator ${ }^{25,26}$.

\section{OFDM as PHY Layer of WiMAX}

The main undertaking of the physical layer is to conceal input details of binary digits into signals and to make them applicable to wireless channel, signals are transmitted and received. This chore is done by the following process as shown below. The block diagram for WiMAX system (802.16e standard) is shown in Figure 1.

\subsection{Randomization}

TFirst and foremost task in WiMAX physical layer where randomization is done to remove copied patterns, like long sequence of nulls and ones. Mainly generates random sequence to improve coding performance.

\subsection{Forward Error Correction (FEC)}

It encodes data and redundant bit stream to permit receiver to observe and correct errors. Coding system involved in FEC are RS codes, TURBO codes, convolution codes etc. It increases the capacity of channel.

\subsubsection{RS codes}

They are symbol error correcting codes. They are a class of non-binary codes having strong error correcting ability.

\subsubsection{Convolution Codes}

It corrects random errors in data transmission. Here every coded bit is a linear combination of some encoded bits. The input is information bits applied to shift register and output is not only a function of current input but also a function of K-1 inputs. Coding rate is defined as ratio of data rates assigned for substitute fram+es to maximum data rate that can perfectly be assigned in substitute frame. The output of convolution encoder is then penetrated to eliminate additional encoded bits and number of bits displaced depends on code rate used.

\subsection{Interleaving}

It is process to make system more efficient fast by arranging data in chaotic manner before transmission. It reorders the data that is to be dispatched so that bytes of data dispensed over large sequence of data to diminish burst errors. The number of bits in each block is known as interleaving gulf, which represents deferment introduced by interleaver at transmitter side. Block interleaver is a type of matrix in which, to read the data, we go for row and switch to column for writing the data and vice versa.

\subsection{Modulation}

After interleaving bits are organized into slots. Due to burst errors, large adjacent bits are corrupted. So, mapping is done. Data bits enter serially to constellation mapper and signal is balanced by digital modulating arrangement; such as M-QAM and M-PSK, where M is a number pattern of points in the constellation diagram and demodulation is done to recover the authentic transmitted digital information.

\subsection{Inverse FFT}

It mainly converts symbols from frequency region to time region and serve OFDM subcarrier as a channel in time 


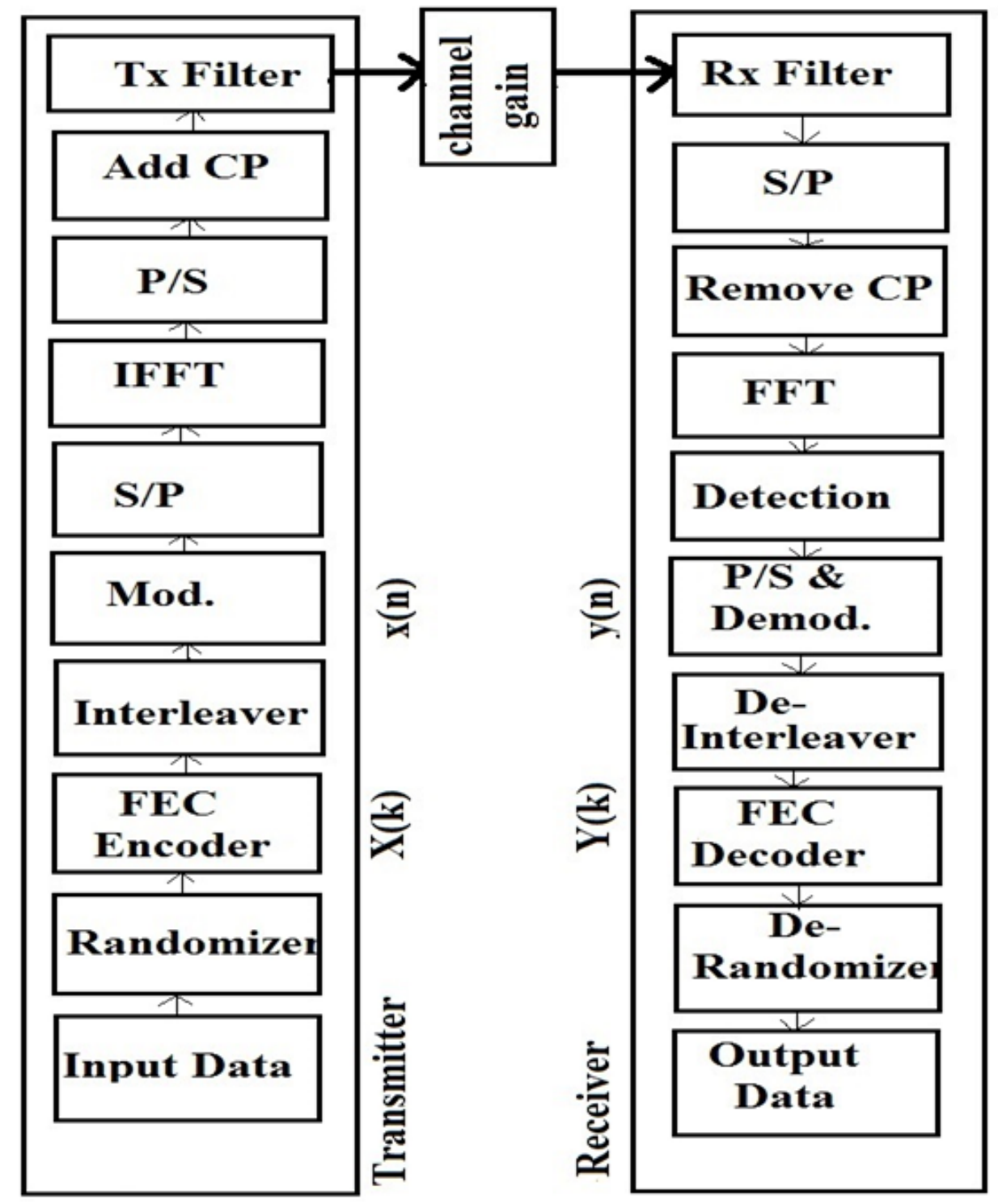

Figure 1. WiMAX physical layer model.

region. If $\mathrm{N}$ no. of subcarriers is used then IFFT receives $\mathrm{N}$ no. of sinusoidal and $\mathrm{N}$ symbols at a time.

\subsection{Cyclic Prefix}

It imitates some samples from the end of the symbol in front to add some tautology to symbols. These cloned samples are called Cyclic Prefix. Main purpose is to avoid inter symbol interference caused by multipath propagation.

\subsection{Reciever}

They are totally opposite of transmitter block. Main purpose is to obtain original data bits. Cyclic prefix is removed as guard interval is removed. Then FFT is per- 
formed and transformed in frequency domain. Then demodulation, de interleaving and de randomization takes place smoothly.

\section{MIMO Systems}

MIMO was presented to satisfy the aspirations for providing reliable great rapid wireless communication connections in severe surroundings. Numerous antennas nas, where sender and receiver send more than one data signal with same radio channel. It mainly has three categories. Beam forming for controlling the inclination to impart and conceal of radio signals. By sending same data to transmitter and receiver so that they experience different fading is spatial diversity. Third category includes spatial multiplexing where different data is set at two different antennas and we get maximum throughput. Some of the MIMO techniques are listed below:

\section{Transmitting antennas}

MIMO-Channels

\section{Receiving antennas}

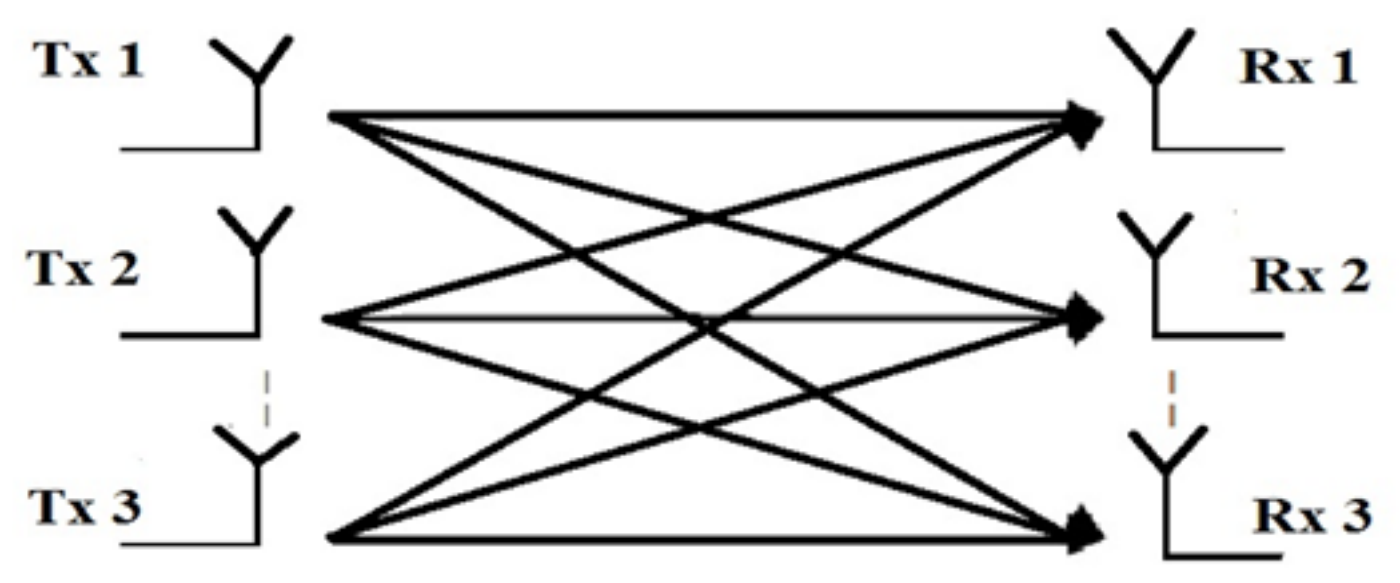

Figure 2. Block diagram of MIMO system.

are used in cellular communication at both transmitter and receiver sides to achieve high data rate through spatial multiplexing. Here multiple transmitters send unique data to various receivers as shown in Figure 2. MIMO requires better SNR and increases data rates along with provide high capacity which is directly proportional to antennas. It mainly consists of three categories. There are already many communication systems is used which occupy available bandwidth and nowadays bandwidth communication is required and using one antenna at both the ends will not work. So, we move for multiple anten-

\subsection{Spatial Diversity (SD)}

A method that provides two or more inputs at the receiver such that inputs of the fading phenomena are not connected. If one radio path undergoes intense fade at certain time, another autonomous path may have a stable signal at that input. Multiple branches and low correlation between the branches are the particular requirements of diversity. All connected properties of the radio channel are used as average to provide multiple independent copies of the same signal. Assuming two antennas at transmit- 
ter side and we use them to send same data. Since these two antennas are set far enough from each other, the same data are transmitted to receiver via different paths that is experiencing different signals called as spatial diversity or transmit diversity. It combats channel fading and provides reliable communications.

\subsection{Spatial Multiplexing (SM)}

Mainly used for high throughput with spatial multiplexing, numerous data streams are transmitted at similar time. They are transmitted on same channel but by various antennas. They are combined at the receiver via MIMO signal processing. Here, data reliability is not fixed. Data rate of the system increases as each spatial channel hoist separate information. And compared to Orthogonal Frequency Division Multiplexing (OFDM) technique, where, different frequency sub channels carry different parts of the adjusted data. But in spatial multiplexing, if there is strong collision, several independent sub channels are generated in the same assigned bandwidth. Thus the compounded growth is useful in both bandwidth and power. It increases channel capacity at high SNR and add complexity and expenses at both transmitter and receiver.

\section{Beamforming}

Beamforming makes use of fading channels and improves gain of received signals and communication system. Beamforming enlarges the performance of mobile communication at average ranges. At short ranges, the SNR will support the maximum data rate because of high signal power. At long ranges, beam forming does not propound a significant gain over a non-directional antenna and data rates will be similar to non-beam formed transmissions. Beamforming makes use of antenna array and to make best use of signal strength, signal processing techniques are used.

\section{MIMO-WiMAX}

WiMAX based companies are trying to have something better for future prospectus in this field using the term MIMO along with it. Using MIMO systems with WiMAX gives better BER performance compared to simple WiMAX. The MIMO schemes under WiMAX technology are determined by space-time coding and spatial multiplexing where each of them performs different functions. WiMAX includes Matrix A and Matrix B MIMO.

\subsection{MATRIX A (Reliable Communication)}

- MATRIX A (Reliable Communication)

- Improves SNR at receiver side.

- Expand cell radius.

- Use of high order modulation to increase transmission speed.

- Better throughput.

\subsection{MATRIX B (Increased Channel Capacity)}

- MATRIX B (Increased Channel Capacity)

- Unconventional data streams over each antenna.

- Perfect signal conditions.

- Use of data rate according to the number of antennas used.

To improve high data rate and spectral efficiency it is more advisable to use MIMO with WiMAX technology as shown in Figure 3. At low BER values, Matrix A has good performance due to expansion in diversity arrangement and Matrix B at SNR values grows the turnout. By using various MIMO schemes in WiMAX system, best BER performance can be maintained through adaptive MIMO switching and by using this, it make use of both MATRIX A and MATRIX B type of modulation. If we simulate modern coding techniques Space Time Coding (STC), Space Frequency Coding (SFC), Space Time Frequency Coding (STFC) on MIMO-WiMAX platform then it contains link between antenna at transmitter and receiver and huge multipath delays. MIMO-WiMAX provides many directions in which independent result can occur and to use them exclusively to find maximum diversity from the channel. 


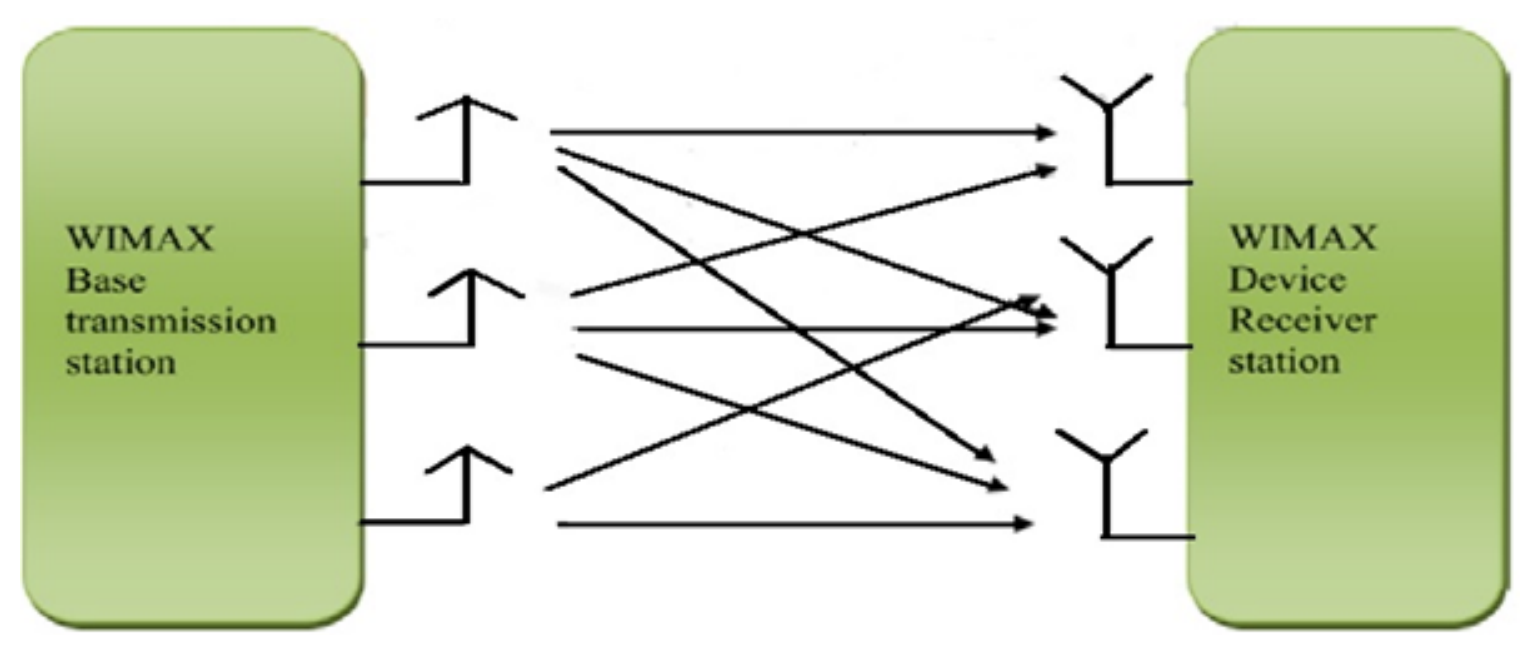

Figure 3. MIMO-WiMAX system communication.

If performance of WiMAX system is estimated by adaptive modulation then there are bigger chances for BER to improve and also it is mostly seen that in frequency selective fading channel is stirred by ISI in huge number as compared to flat fading. Researchers tried to relay out WiMAX simulations in OPNET which worked very well but it was not capable to take MIMO technique along with it. Companies like Huawei, Intel, etc. are bound in finding better solutions for MIMO based WiMAX systems. Best example is WiBro network in Korea from where Tikona Digital Networks from India offers WiBro service for up to 2 Mbits/sec. WiMAX ratify MIMO antenna strategy which shows accompaniment in terms of spectral efficiency and link reliability. Performance of MIMOWiMAX systems in a multicell, multisector and multiuser surroundings is of great impact in WiMAX technology by using single frequency.

\section{ADVANTAGES}

- Meets the demand of LoS (Line of Sight) in microwave communications against NLOS and multi fade paths.
- Increase spectral efficiency compared to SISO systems.

- Flexible sub-channelization and Adaptive Modulation and Coding (AMC) enables MIMOWiMAX technology to improve system coverage, capacity, better BER, throughput and efficiency.

\section{Conclusion}

WiMAX is based on Broadband Wireless Access (BWA). The stipulation for high data rate wireless communication has been increasing drastically. In this paper we have discussed about the basic idea behind the OFDM, the most emerging technology of this era. Here we take a review on MIMO systems, its properties and discussed about its techniques and tactics used by authors in describing the best methods to cure problems of WiMAX along with use of this in various algorithms, formulas, diagrams and much more. It helps us in finding better perspectives for upcoming technologies and for upgradation of imminent generation and country. Highlighting the combination of MIMO along with WiMAX and their consequences by explaining its role in removing multipath fading channels, 
having better capacity, coverage, throughput, efficiency and BER. This paper has explored the role of WiMAX PHY layer in the wireless communication.

\section{References}

1. Rohling M, May T, Bruninghaus K, Grunheid R. Broad-band OFDM radio transmission for multimedia applications. Proceedings of the IEEE. 1999 Oct; 87(10):1778-89.

2. Chen B, Wang H. Maximum likelihood estimation of OFDM carrier frequency offset. IEEE International Conference on Communications; 2002 Apr. p. 49-53.

3. Banelli P, Cacopardi S. Theoretical analysis and performance of OFDM signals in nonlinear AWGN channels. IEEE Transactions on Communications. 2000 Mar; 48(3):430-41.

4. Murty MS, Veeraiah D, Rao AS. Performance evaluation of Wi-Fi comparison with WiMAX Networks. International Journal of Distributed and Parallel Systems. 2012 Jan; 3(1):321-8.

5. Tilwari V, Kushwah AS. Performance analysis of WiMAX 802.16e physical layer using digital modulation techniques and code rates. International Journal of Engineering Research and Applications. 2013 Jul; 3(4):1449-54. 22489622.

6. Agarwal A, Mehta SN. Combined effect of block interleaving and FEC on BER performance of OFDM based WiMAX (IEEE 802.16 d) System. American Journal of Electrical and Electronic Engineering. 2015; 3(1):4-12.

7. Divya S, Kumar HA, Vishalakshi A. An improved spectral efficiency of WiMAX using 802.16G based technology. 2015 International Conference on Advanced Computing and Communication Systems; 2015 Jan. p. 1-4.

8. Bighieri E, Goldsmith A, Muquet B, Sari H. Diversity, interference cancellation and spatial multiplexing in MIMO mobile WiMAX systems. 2007 IEEE Mobile WiMAX Symposium; 2007 Mar. p. 74-9.

9. Peppas K, Lazarakis F, Axiotis DI, Al-Gizawi T, Alexandridis AA. System level performance evaluation of MIMO and SISO OFDM-based WLANs. Wireless Networks. 2009 Oct; 15(7):859-73.

10. Li Q, Lin XE, Zhang J. MIMO precoding in $802.16 \mathrm{e}$ WiMAX. Journal of Communications and Networks. 2007 Jun; 9(2):141-9.
11. Tan K, Andrian JH, Zhu H, Candocia FM, Zhou C. A novel spectrum encoding MIMO communication system. Wireless Personal Communications. 2010 Jan; 52(1):14763.

12. Hemalatha M, Prithviraj V, Jayalalitha S, Thenmozhi K. Space diversity knotted with WiMAX - A way for undistorted and anti-corruptive channel. Wireless Personal Communications. 2013 Aug; 71(4):3023-32.

13. Alex SP, Jalloul LMA. Performance evaluation of MIMO in IEEE 802.16e/WiMAX. IEEE Journal of Selected Topics in Signal Processing. 2008 Apr; 2(2):181-90.

14. Sezginer S, Sari H, Biglieri E. A comparison of full-rate fulldiversity $2 \times 2$ space-time codes for WiMAX systems. 2008 IEEE 10th International Symposium on Spread Spectrum Techniques and Applications; 2008 Aug. p. 97-102.

15. Zerrouki H, Feham M. A physical layer simulation for WiMAX MIMO-OFDM system: Throughput comparison between $2 \times 2$ STBC and $2 \times 2 \mathrm{~V}$-BLAST in Rayleigh fading channel. 2014 International Conference on Multimedia Computing and Systems; 2014 Apr. p. 757-64.

16. Farhan F. Study of timing synchronization in MIMOOFDM systems using DVB-T. International Journal on Information Technology. 2014 Apr; 3(2):1-10.

17. Pachori K, Mishra A. An efficient combinational approach for PAPR reduction in MIMO-OFDM system. Wireless Networks. 2016 Feb; 22(2):417-25.

18. Panchal AK. Performance evaluation of MIMO-OFDM schemes in presence of Carrier Frequency Offset (CFO). International Journal of Innovative Research in Electrical, Electronics, Instrumentation and Control Engineering. 2015 Apr; 3(4):100-3.

19. Mare KP, Maharaj BT. Performance analysis of modern space-time codes on a MIMO-WiMAX Platform. 2008 IEEE International Conference on Wireless and Mobile Computing, Networking and Communications; 2008 Oct. p. $139-44$.

20. Kobeissi R, Sezginer S, Buda F. Downlink performance analysis of full-rate STCs in 2x2 MIMO WiMAX systems. IEEE 69th Vehicular Technology Conference; 2009 Apr. p. $1-5$.

21. Muquet B, Biglieri E, Sari H. MIMO link adaptation in mobile WiMAX systems. 2007 IEEE Wireless Communications and Networking Conference; 2007 Mar. p. $1810-3$. 
22. Mehlfuhrer C, Caban S, Garcia-Naya JA, Rupp M. Throughput and capacity of MIMO WiMAX. 2009 Conference Record of the Forty-Third Asilomar Conference on Signals, Systems and Computers; 2009 Nov. p. 1426-30.

23. Kalaivani D, Karthikeyen S. VLSI implementation of areaefficient and low power OFDM transmitter and receiver. Indian Journal of Science and Technology, 2015 Aug; 8(18). DOI: $10.17485 /$ ijst/2015/v8i18/63062.

24. Avila J, Thenmozhi K. Let Multiband-OFDM modified by wavelet. Indian Journal of Science and Technology. 2014 Jan; 7(8). DOI: 10.17485/ijst/2014/v7i8/42090.
25. Manikandan C, Neelamegam P, Divya E. OFDM techniques for MIMO-OFDM system: A review. Indian Journal of Science and Technology. 2015 Sep; 8(22). DOI: 10.17485/ ijst/2015/v8i22/79103.

26. Kasiselvanathan M, Sathish Kumar N. BER performance analysis and comparison for large scale MIMO receiver. Indian Journal of Science and Technology. 2015 Dec; 8(35). DOI: 10.17485/ijst/2015/v8i35/81073. 